\title{
VSOP and VLBA observations of EGRET-identified Active Galactic Nuclei
}

\section{Kiyoaki Wajima*}

KVN Group, Korea Astronomy and Space Science Institute

E-mail: kiyoaki@trao.re.kn

\begin{abstract}
We made high-resolution VLBI observations of six southern gamma-ray loud quasars, which were identified by EGRET (Energetic Gamma-Ray Experiment Telescope) on-board gamma-ray detector, with VSOP (VLBI Space Observatory Programme) and the VLBA. All sources show a compact core-dominant structure and the core component of three sources has a brightness temperature greater than $10^{12} \mathrm{~K}$ in the source's rest frame. Having combined our observations with previous results we conclude that two sources out of three (PKS 0336-019 and PKS 0537-441) have small jet viewing angles. Based on two-epoch VSOP observations, we found that also the third source, PKS 1741-038, has the jet viewing angle smaller than $4.8^{\circ}$. On the other hand, an observation of PKS 1622-297, exhibiting a spectacular GeV gamma-ray flare, shows rather lower Doppler factor and larger jet viewing angle than those of other gamma-ray-loud active galactic nuclei (AGNs). We examine the gamma-ray emission mechanism in the light of these observations.
\end{abstract}

The 8th European VLBI Network Symposium

September 26-29, 2006

Toruń, Poland

${ }^{*}$ Speaker. 


\section{Introduction}

Observations made with the EGRET gamma-ray detector on-board the CGRO spacecraft resulted in the identification of nearly 70 AGNs emitting gamma-rays [1]. Recent VLBI observations have revealed several features of the parsec-scale structures in gamma-ray-loud AGNs, such as smaller jet viewing angles and higher Doppler factors. There are, however, still large uncertainties of the physical parameters in gamma-ray-loud AGNs and it is, therefore, important to verify precisely the milliarcsecond (mas)-scale properties of individual sources by VLBI observations.

Here, we report the mas-scale structure of six southern EGRET-identified AGNs with the VLBA and VSOP [2]. VSOP observation can attain a much better angular resolution and a $u-v$ coverage even for southern sources by choosing a favourable observing epoch. Longer baselines compared to ground-based VLBI also enable us to make precise measurement of the lower limit of the brightness temperature.

\section{Observations, results and discussion}

Observations were made from August 1997 through September 1999 by VSOP with $3-12$ ground radio telescopes out of 17 stations including the VLBA. We carried out a priori amplitude calibration and fringe-fitting using AIPS. Imaging and model fitting were carried out using Difmap.

Figure 11 shows the images of the observed sources. All sources have compact core-dominant structures with weak jet(-like) components. To quantify the source size and to estimate the source brightness temperature we modeled the calibrated visibility data with elliptical Gaussian components. Table 1 gives the model fitting results for the core component of each source. From those parameters, we can calculate the source brightness temperature $\left(T_{\mathrm{B}}\right)$ in the source's rest frame. The results are also shown in Table 1. The core component of three sources has $T_{\mathrm{B}}$ of greater than

Table 1: Model fitting results.

\begin{tabular}{cccccccc}
\hline \hline Source & Redshift & Date & $\begin{array}{c}v^{*} \\
{[\mathrm{GHz}]}\end{array}$ & $\begin{array}{c}S^{\dagger} \\
{[\mathrm{Jy}]}\end{array}$ & $\begin{array}{c}\theta_{\text {maj }} \| \\
{[\mathrm{mas}]}\end{array}$ & Ratio $^{\#}$ & $\begin{array}{c}T_{\mathrm{B}}^{\S} \\
{\left[\times 10^{11} \mathrm{~K}\right]}\end{array}$ \\
\hline PKS 0336-019 & 0.852 & 1999 Feb 6 & 5 & 1.44 & 0.38 & 0.43 & 21.8 \\
PKS 0537-441 & 0.894 & 1999 Sep 4 & 5 & 3.18 & 0.37 & 0.56 & 39.9 \\
PKS 1510-089 & 0.361 & 1997 Aug 13 & 1.6 & 1.77 & 2.62 & 0.28 & 5.7 \\
PKS 1622-297 & 0.815 & 1998 Feb 22 & 5 & 1.50 & 0.71 & 0.76 & 3.7 \\
PKS 1741-038 & 1.054 & 1997 Jul 5 & 1.6 & 1.93 & 1.06 & 0.80 & 20.2 \\
& & 1997 Jul 5 & 5 & 4.23 & 0.48 & 0.69 & 27.7 \\
PKS 1933-400 & 0.966 & 1998 Apr 6 & 5 & 3.04 & 0.35 & 0.75 & 34.5 \\
\hline
\end{tabular}

Results for the core component are shown.

* Observation frequency. ${ }^{\dagger}$ Flux density of the core component. " , \# Major axis and ratio of minor to major axes of Gaussian model. ${ }^{\S}$ Peak brightness temperature given in the source's rest frame. 

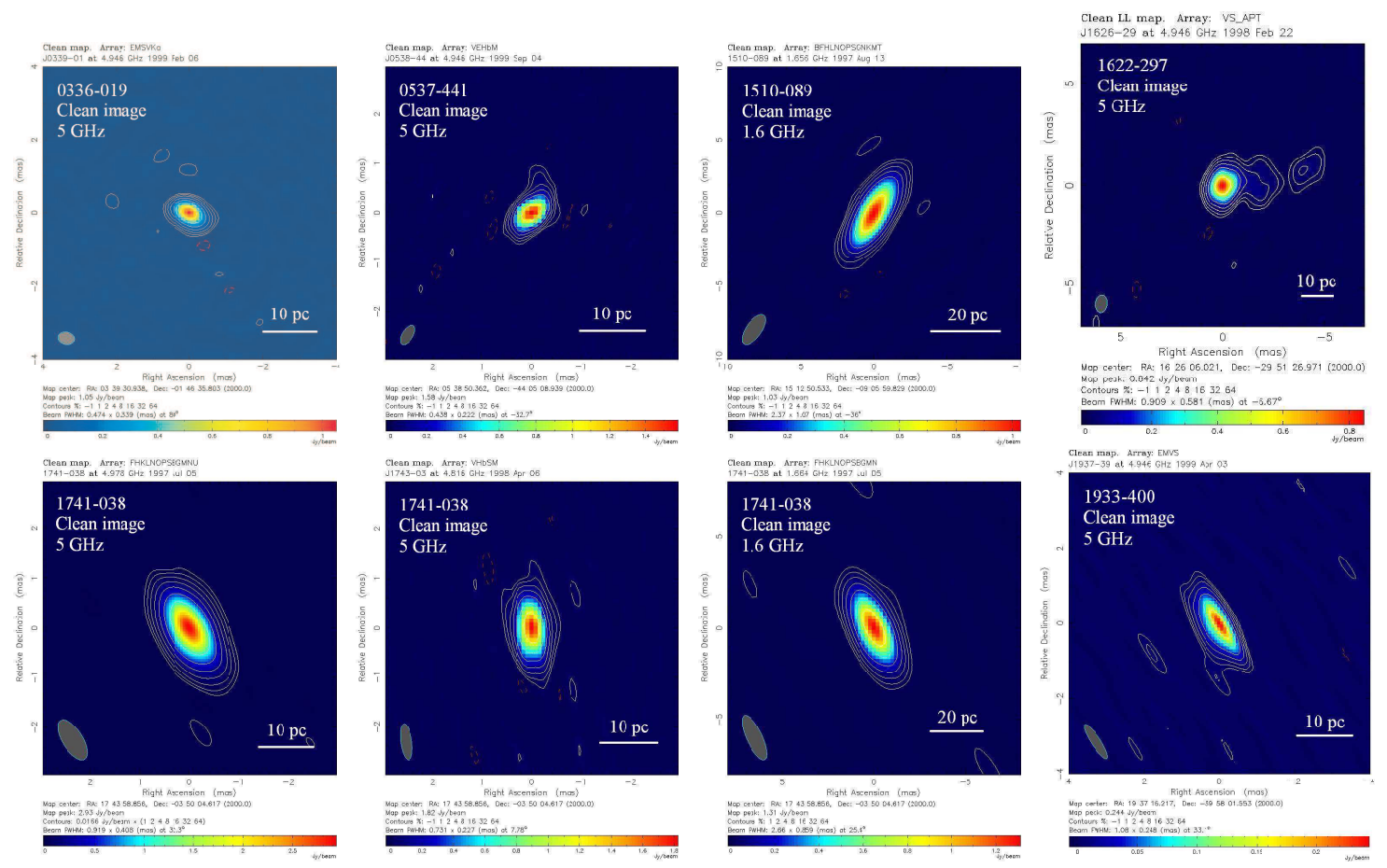

Figure 1: Images of each source. The contour levels are $\left(-1,2^{n}\right) \times$ the peak intensity $(n=0,1, \cdots, 6)$.

$10^{12} \mathrm{~K}$. We also found that one of three sources, PKS 1741-038, has small jet viewing angle $\left(\phi<4.8^{\circ}\right)$ based on two-epoch VSOP observations. [3]. Having combined our observations with previous results, we conclude that other two sources, PKS 0336-019 and PKS 0537-441, also have small viewing angles $\left(\phi \leq 5^{\circ}\right)$. Those results favour the inverse Compton gamma-ray emission model and we conclude that the gamma-ray emission from them is highly Doppler boosted.

On the other hand, the results with a detailed analysis of the observation of PKS 1622-297 show rather lower Doppler factor $(\delta \sim 2.3)$ and larger jet viewing angle $\left(\phi \sim 23.3^{\circ}\right)$ for the inner component [ [4]. These features are rather different from other gamma-ray-loud AGNs. Based on our observational results, we cannot assert that gamma-ray emission from this source is caused by inverse Compton scattering by relativistic electrons in the parsec-scale jet. Further VLBI observations of this source are needed and further consideration as to whether it is possible to detect significant gamma-ray emission from AGNs having such large viewing angle is required.

\section{Acknowledgments}

We gratefully acknowledge the VSOP Project, which is led by the Institute of Space and Astronautical Science of Japan Aerospace Exploration Agency in cooperation with many organizations and radio telescopes around the world. The VLBA is the facility operated by Associated Universities Inc. under agreement with the National Science Foundation. 


\section{References}

[1] R. C. Hartman, et al., The Third EGRET Catalog of High-Energy Gamma-Ray Sources, ApJS 123 (1999) 79.

[2] H. Hirabayashi, et al., Overview and Initial Results of the Very Long Baseline Interferometry Space Observatory Programme, Science 281 (1998) 1825.

[3] K. Wajima, et al., Two-Epoch Space VLBI Observations of the Gamma-Ray Loud Quasar PKS 1741-038, PASJ 52 (2000) 329.

[4] K. Wajima, et al., Milliarcsecond-Scale Structure in the Gamma-Ray Loud Quasar PKS 1622-297, PASJ 58 (2006) 223 [astro-ph/ 0511063 ]. 